\title{
Total hip arthroplasty in patients with severe hip dysplasia and congenital pubic diastasis: report of two cases
}

\author{
Christian Goetze ${ }^{1}$, Filippo Migliorini ${ }^{2 *}$ and Christian Dominik Peterlein ${ }^{1}$
}

\begin{abstract}
Background: Congenital bladder exstrophy is a rare malformation which is often associated with pubic diastasis and hip dysplasia. We reported the case two patients who underwent total hip arthroplasty (THA) due to advanced osteoarthritis combined with large congenital pubic diastasis $(>10 \mathrm{~cm})$.

Case presentation: The first patient, a 39 years old woman with a pubic diastase and severe hip dysplasia on both sides was treated with a primary two-staged bilateral THA. Both hips were treated with a cementless osteoconductive cup (TM, Zimmer-Biomet) and a cementless stem (Alloclassic SL, Zimmer-Biomet). A $10^{\circ}$ elevated rim liner of the cup was used in order to avoid dislocation. The main problem was represented by the fixation of the cup, given the retroverted acetabulum along with the elevated rotation centre due to the dysplastic hips. In the case two, a 52 years woman presented dysplastic osteoarthritis of the left hip. A conventional hemispherical cup (Alloclassic-Allofit, Zimmer-Biomet) was placed in the retroverted acetabulum combined with a cementless stem (Fitmore A, ZimmerBiomet) attached at the metaphyseal proximal femur bone.
\end{abstract}

Conclusion: Our results suggest that THA may be a good strategy to manage advanced hip osteoarthritis in patients with dysplasia and congenital pubic diastasis.

Level of evidence: $\mathrm{N}$, case series.

Keywords: Congenital pubic diastasis, Dysplasia, Arthroplasty, Bladder exstrophy

\section{Background}

Bladder exstrophy is rare malformation belonging to the inferior coelosomies [1]. These malformations are characterized by an incomplete closure of the lower abdominal wall during the embryonic development [2]. Bladder exstrophy has a prevalence of approximately one over 30,000 newborns, with higher prevalence in girls [3]. Bladder exstrophy is commonly associated with a wide range of genitourinary, musculoskeletal, and intestinal malformations [4]. Among the musculoskeletal abnormalities,

*Correspondence: migliorini.md@gmail.com

2 Department of Orthopaedics, Trauma, and Reconstructive Surgery, RWTH University Hospital, Pauwelsstr. 30, 52074 Aachen, Germany

Full list of author information is available at the end of the article symphysis diastasis, hip dysplasia, and rotational abnormalities (e.g. external rotation of the posterior pelvis and iliac wings, acetabular retroversion) are common [5-8]. Shortening of the pubic bones is also common $[9,10]$. A prompt closure of the pubic diastasis avoids degeneration of the inner membrane of the bladder [11]. To achieve the closure a direct suture is preferred [4]. Osteotomies are deserved for large diastases: external iliac osteotomy of the sacroiliac joint followed by internal bending of the ileum; osteotomy of the proximal end of the greater sciatic notch followed by internal rotation of the distal bone section; osteotomy of the lateral pubis followed by approximation; dorsal and coronal osteotomy laterally to the sacroiliac joints and distal to the sciatic notch, 
positioning of bone fragments, and fixation of the pubic bones with a metal plate [4, 12-14].

Little is known about patients who underwent bladder exstrophy repair without pelvic diastasis closure. Retroversion of the dysplastic acetabulum, rotation of the posterior part of the pelvis and iliac wings, and instability of the pelvic ring are the most challenging aspects which the orthopaedic surgeon must be aware when facing these patients $[15,16]$. Hence, we presented a case series of two adults who underwent surgical bladder exstrophy repair but with neglected closure of pelvis diastasis. Both patients presented severe coxarthrosis due to dysplasia and underwent total hip arthroplasty (THA).

\section{Case presentation Case one}

A 35 years old woman with a BMI of $46,7 \mathrm{~kg} / \mathrm{m}^{2}$ was admitted at our department with severe pain in both hips. She underwent multiple surgical interventions to reduce bladder exstrophy. An external urinary kidney catheter was in place. The patient used a wheelchair even for shorter distances. Mobilization waddling gait with crutches shorter than $10 \mathrm{~m}$. The range of motion of both hips were considerably impaired (Right: ext./ flex $0^{\circ} / 0^{\circ} / 70^{\circ}$, internal/external rotation in $70^{\circ}$ flexion: $0^{\circ} / 0^{\circ} / 10^{\circ}$; abduction adduction $20^{\circ} / 0^{\circ} / 10^{\circ}$. Left: ext./ flex $0^{\circ} / 0^{\circ} / 60^{\circ}$, internal/external rotation in $70^{\circ}$ flexion: $0^{\circ} / 0^{\circ} / 5^{\circ}$, abduction adduction: $10^{\circ} / 0^{\circ} / 10^{\circ}$ ). Radiographies (Fig. 1) demonstrated severe bilateral hip osteoarthritis grade III, according to the Tönnis classification [17], grade II femoral head dislocation, according to the Crowe

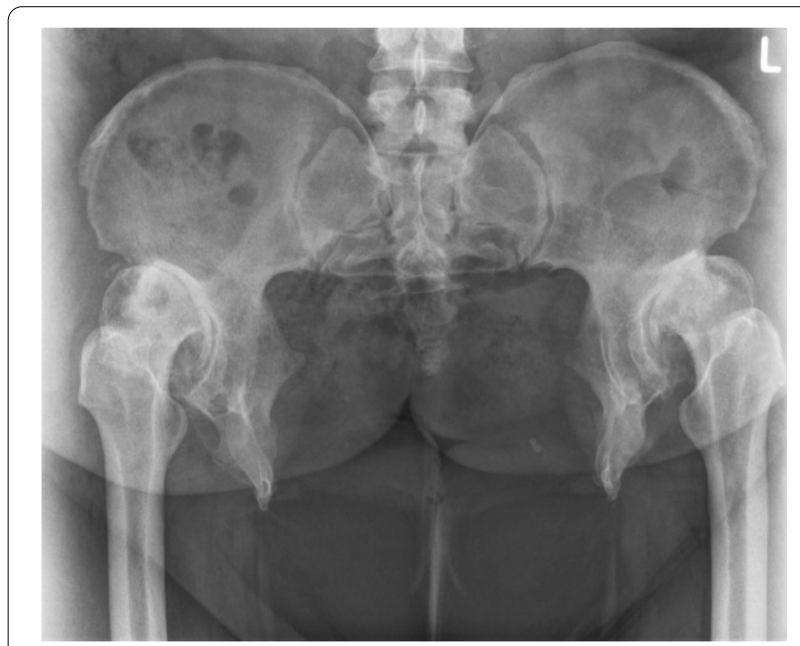

Fig. 1 Antero-posterior radiography of the pelvis which shows advanced osteoarthritis secondary to hip dysplasia of both sides combined with a congenital pubic diastasis of $18 \mathrm{~cm}$ classification [18], $18 \mathrm{~cm}$ width pubic diastasis, and shortening of the anterior ischiopubic segment.

Computed tomography (CT) (Fig. 2) showed increased external rotation of the posterior pelvis and iliac wings, combined with $12^{\circ}$ right and $2^{\circ}$ left acetabular retroversion. In 2011 a two-staged THA was performed, with 3 months interval between the two interventions.

The senior author (C.G.) did both operations. A lateral approach with in supine position was used. An osteoconductive cup with high porosity to enhance primary fixation and optimized secondary osteointegration (Trabecular Metal $^{\mathrm{TM}}$ modular cup, Zimmer-Biomet $\mathrm{GmbH}$, Winterthur, size $56 \mathrm{~mm}$ ) was placed with an additional anteverted liner $20^{\circ}$ to maintain stability and prevent dislocation (Fig. 3a). Two screws were tightened to increase primary stability of the cementless cup on the right side, while none was required in the left hip (Fig. 3b). On the femoral side a distally-fixed stem Alloclassic-SL (Zimmer-Biomet $\mathrm{GmbH}$, Winterthur) was used to reconstruct anteversion of the proximal femur.

Average surgical duration was $75 \mathrm{~min}$, the intraoperative blood loss was $300 \mathrm{ml}$. No drains were used. The post-operative rehabilitation started $24 \mathrm{~h}$ post-operatively. Full weightbearing was allowed. Passive and active hip motion started at $48 \mathrm{~h}$ postoperatively. From the third postoperative day, patients were allowed to stand, and walking re-education program starts with a fully trained physiotherapist. The patient was discarded at sixth postoperative day in a rehabilitation clinic. After 3 months from the last THA, the patient presented had no pain, declaring herself very satisfied with the result. The walk distance with one left crutch increased to $200 \mathrm{~m}$. Similar results were found at last follow-up at 9 years.

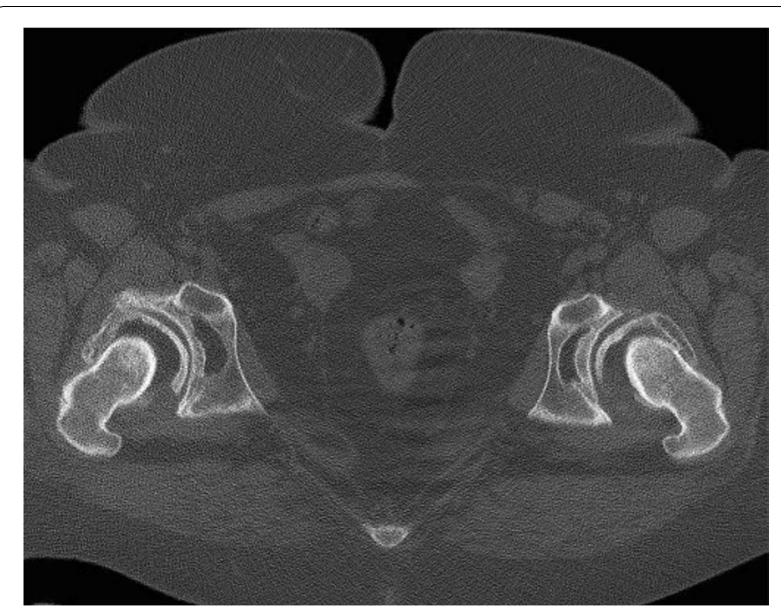

Fig. 2 Sequence of the computed tomography of the pelvis which shows the acetabular retroversion combined with external rotation of the posterior pelvis and iliac crests 


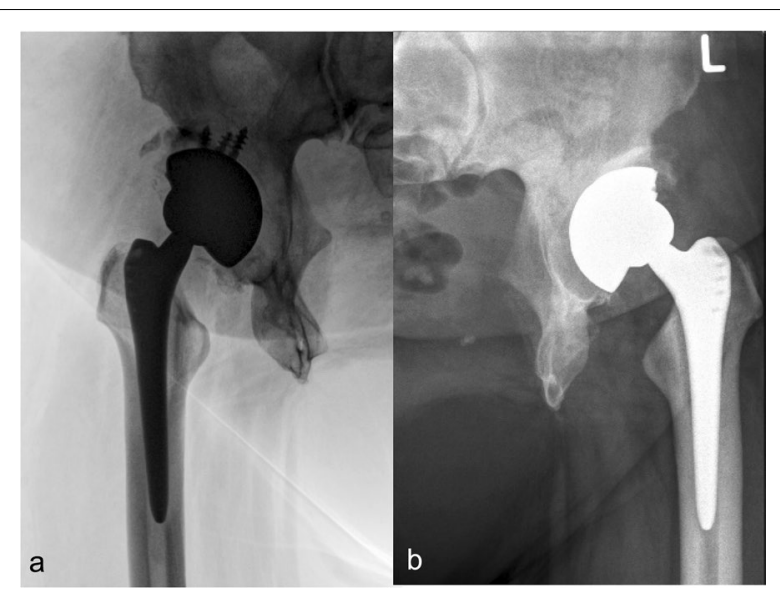

Fig. 3 a) Postoperative radiography of the right hip with the osteoconductive cup (TM modular cup) size $56 \mathrm{~mm}$ with an additional anteverted liner $20^{\circ}$ combined with distally fixed stem (Alloclassic-SL); b) Postoperative radiography on the left hip at 3 months

Radiographic control evidenced no sign of loosening of the components or heterotopic ossification (Fig. 4a, b). The Harris hip Score [19] increased from 14 points preoperatively to 68 at 9 years follow-up. The postoperative

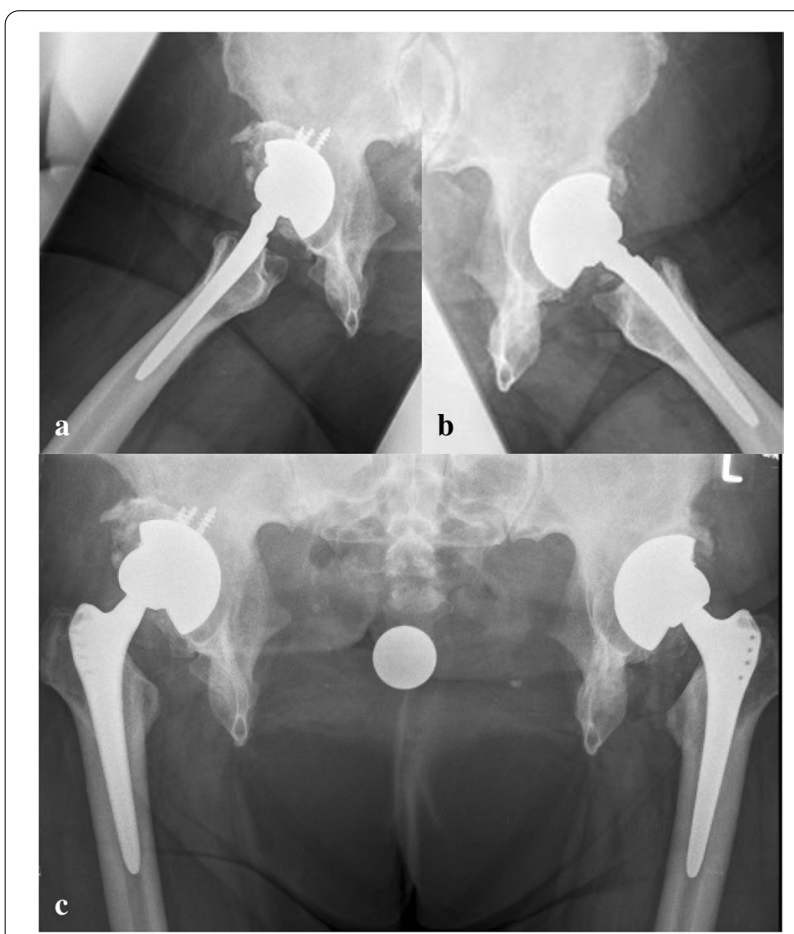

Fig. 4 a, b) 9 years postoperative radiographic control demonstrated no signs of loosening of the acetabular or femoral component; c) 9 years postoperative radiographic control in lateral approach evidenced no signs of loosening of the implants right cup inclination and anteversion were $67.3^{\circ}$ and $0^{\circ}$, respectively. At last follow-up, both inclination and anteversion remained almost unchanged $\left(67.5^{\circ}\right.$ and $0^{\circ}$, respectively). Similarly, also the left cup remained unchanged (inclination $69.1^{\circ}$ versus $69.2^{\circ}$; anteversion $0^{\circ}$ versus $0^{\circ}$ ). No difference was found in the width of pubic diastasis post-operatively to last follow-up $(18.0$ to $18.1 \mathrm{~cm})$. No complication was experienced by the patient.

\section{Case two}

The second case was a 52 years old woman with prior multiple abdominal operations and a pubic catheter due to congenital bladder aplasia. The patient worked at the time of the surgery as anaesthetist, and declared herself satisfied with work activity and quality of life. The only reason for seeking orthopaedic advice was increased left hip pain and decreased walking distance. The range of motion was considerably impaired (ext./flex: $10^{\circ} / 0^{\circ} / 80^{\circ}$; internal/external rotation in $70^{\circ}$ flexion: $10^{\circ} / 0^{\circ} / 15^{\circ}$; abduction adduction: $\left.30^{\circ} / 0^{\circ} / 15^{\circ}\right)$. Radiographically advanced osteoarthritis due to severe dysplasia combined with a congenital pubic diastasis of $14.6 \mathrm{~cm}$ (Figs. 5 and 6) was evidenced.

Computed tomography (CT) (Fig.6) showed increased external rotation of the posterior pelvis and iliac wings, combined with $18^{\circ}$ right and $3^{\circ}$ left acetabular retroversion.

The senior author (C.G.) did the THA. A lateral approach with patient in supine position was used. Average surgical duration was $57 \mathrm{~min}$, the average total intraoperative blood loss was $200 \mathrm{ml}$. A cementless conventional cup (Allofit, Zimmer-Biomet $\mathrm{GmbH}$,

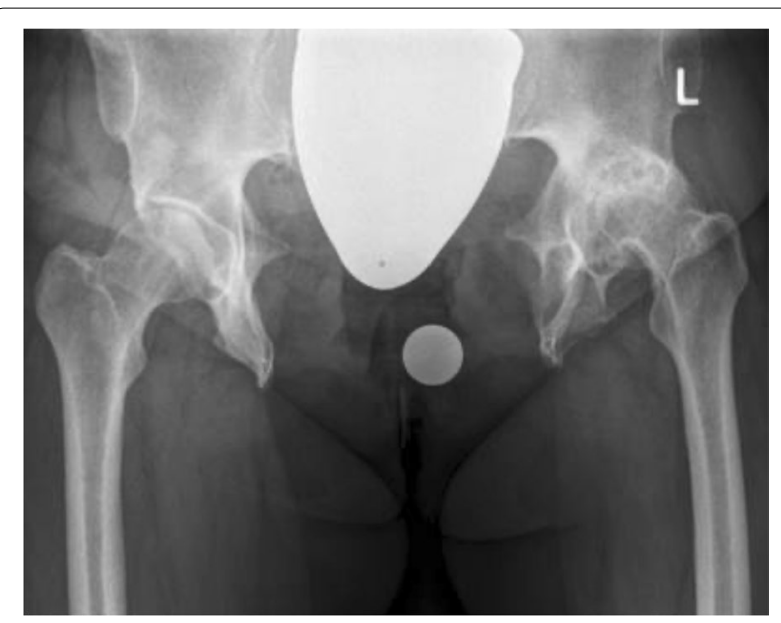

Fig. 5 Antero-posterior radiography of the pelvis which shows advanced osteoarthritis secondary to hip dysplasia combined with a congenital pubic diastasis of $14.6 \mathrm{~cm}$ 


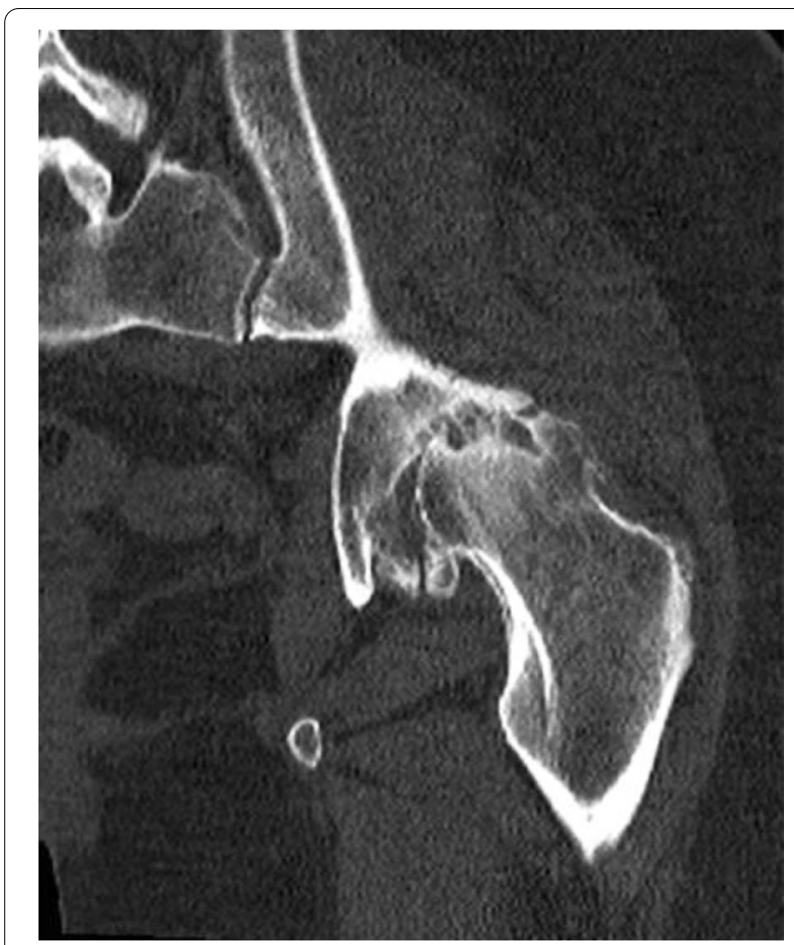

Fig. 6 Sequence of the computed tomography of the pelvis which shows the acetabular retroversion combined with external rotation of the posterior pelvis and iliac crests

Winterthur) with a metaphyseal fitted stem (Fitmore, Zimmer GmbH, Winterthur) was performed (Fig. 7).

No drains were used. The post-operative rehabilitation started $24 \mathrm{~h}$ post-operatively. Full weightbearing was allowed. Passive and active hip motion started at $48 \mathrm{~h}$ postoperatively. From the third postoperative day patients were allowed to stand, and walking re-education program starts with a fully trained physiotherapist. The patient was discarded at 8 days after the procedure in a rehabilitation clinic. At 6 months, the patient has no pain in the operated hip, declaring herself satisfied with the operation. Walking distance was unlimited and she could return to work without any impairment. The Harris Hip Score improved from 40 points preoperatively to 80 at last follow-up. At last follow-up, both cup inclination and anteversion remained almost unchanged $\left(+1.0^{\circ}\right.$ and $+0.5^{\circ}$, respectively). No difference was found in the width of pubic diastasis post-operatively to last followup $(0.1 \mathrm{~cm})$. No complication was experienced by the patient.

\section{Discussion and conclusion}

Congenital pubic diastasis combined with bladder exstrophy is an embryologic malformation which results in a complex deficit of the anterior midline. The improper

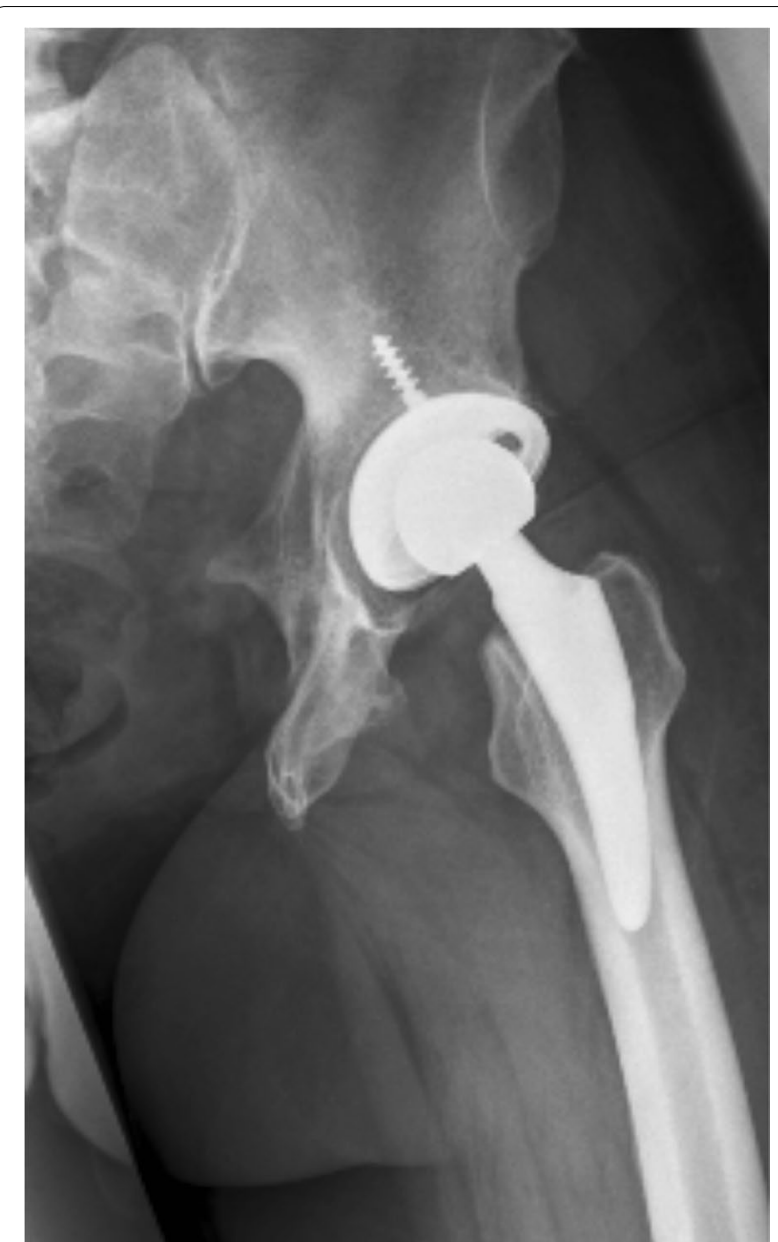

Fig. 7 5-days postoperative antero-posterior radiography of the pelvis which shows conventional cup (Allofit) with a metaphyseal fitted stem (Fitmore)

development of the inner abdominal wall led to prenatally fascia rupture, causing the skeletal and urogenital malformations. In severe cases, the intestinal system is affected with cloacal malformation, in which the terminal part of the rectum ends in the urethra. Along with pelvis diastasis, many patients present associated rotational anomalies, shifting dorsally the axes of the sacrum and ileum. Furthermore, the posterior rotation of the acetabulum also affects the biomechanics of gait and weights distribution, leading in some cases to early onset osteoarthritis. Currently, various procedures to correct the skeletal malformation were described [12-14]. However, these osteotomies are typically performed during early childhood $[8,20]$. In skeletally mature patients ischiopubic osteotomy to correct the diastasis is not possible. This opens concerns regarding the management of these patients, in which diastasis is still present in the adult age. Our results suggested that patients 
with advanced hip osteoarthritis due to dysplasia combined with congenital pubic diastasis may benefit from THA. Moreover, patients maintain a satisfying outcome over the years, almost comparable with patients without diastasis. Finally, no enlargement of the pubic diastasis was detected, and the position of the cup remained unchanged at last follow-up.

The natural history of this malformation, from an orthopaedic point of view, seems to not affect substantially the quality of life and activities daily living. However, given the limitation of this study, these hypotheses are not fully generalizable. We were able to identify only two studies which described the outcomes of patients with congenital pubic diastasis treated with THA [15, 16]. Both studies reported comparable outcomes, recommending THA as treatment for these patients. However, they also included a limited number of procedures and potentially biased results.

Given the lack of the anterior pubis symphysis, along with the retroverted high-angle and stretched acetabulum, these patients presented high pelvic instability. This instability can affect implant survivorship and increase the risk of failure and dislocations. The severe dysplasia of the retroverted acetabular grove in both cases represents the most challenging problem to overcome surgically. In our cohort of patients, the acetabulum presented enough depth to ensure cup components stabilization. The retroverted acetabulum can be compensated by a neutral position of the acetabular cup with elevated liner to avoid femoral head dislocation, while the anterior rim reduction helps preventing impingement. Greater acetabular retroversion, pelvis extrarotation, and insufficient acetabular depth may stress acetabular component fixation. CT helps to investigate the degree of retroversion of iliac crests, acetabular malorientation, and depth of the acetabular cavity. Moreover, CT helps to predict the position of the acetabular components and to plan the restoration of the hip rotating centre in the acetabular groove [21]. In the first case we used an osteoconductive trabecular metal cups to enhance stability [22]. In the late 90's first biomechanical testing of osteoconductive trabecular metal cups presented a high biomechanical stability for cancellous and cortical bone structures [23]. Primary stability and secondary osteointegration will be enhanced by osteoconductive trabecular metal cups, reducing the contact with the acetabular bony cavity [24]. An anteverted liner can be placed to reduce the risk of dislocation, allowing to place the cup with a greater inclination. On the femoral side, given its to distal fixation characteristics, a straight rectangle stem was used. The anteversion of the femoral side can be corrected with regard to the position of the stem. The second case had better position of the acetabulum and proximal femur geometry. A conventional cup with screw fixation presented enough stability for secondary osteointegration. Overall, the short and long-term results were successful. The key success might be the reconstruction of hip rotation centre with the acetabular component. In both cases anterior rim trimming was necessary to avoid THA impingement.

This study has certainly limitations. First, the limited study size which jeopardises the ability to identify important variables leading to poor outcomes. However, it should be noted that these patients are uncommon in the clinical practice. In this respect the results are not fully generalisable. Allocation was highly biased, and the lack of randomization and blinding design also affected negatively the reliability of our conclusions. Thus, data from the present study must be interpreted with caution. These results encourage future research to overcome current limitations, establishing in larger scale potential benefits of THA on these patients, giving lights of recommendations and possible pitfalls.

Concluding, our results suggest that THA may represent a strategy to manage advanced hip osteoarthritis in patients with dysplasia and congenital pubic diastasis. These results must be considered within the limitations of the present study, and further researches are required to overcome current limitations, establishing in larger scale potential benefits of THA on these patients, giving lights of recommendations and possible pitfalls.

\section{Abbreviations \\ THA: Total hip arthroplasty; CT: Computed tomography; TM: Trabecular metal; BMI: Body mass index.}

\section{Acknowledgements \\ None.}

Authors' contributions

CG: surgical procedures, writing and editing; FM: writing and editing; PCD: writing and editing. All authors have read and approved the manuscript.

\section{Authors' information}

Prof. Dr. med. Goetze Christian MD, Department of Orthopaedics, AugusteViktoria Clinic, Ruhr University Bochum, 32545 Bad Oeynhausen, Germany; Dr. med. Migliorini Filippo MD, PhD, MBA, Department of Orthopaedic, Trauma, and Reconstructive Surgery, RWTH University Hospital, 52074 Aachen, Germany; Prof. Dr. med. Peterlein Christian Dominik MD, Department of Orthopaedics, Auguste-Viktoria Clinic, Ruhr University Bochum, 32545 Bad Oeynhausen, Germany.

Funding

Open Access funding enabled and organized by Projekt DEAL.

Availability of data and materials

All data generated or analysed during this study are included in this published article.

\section{Declarations}

Ethics approval and consent to participate

This study complies with ethical standard (Ethic Commission of the Medical Faculty of the Ruhr University of Bochum, Germany). 


\section{Consent for publication}

Written informed consent was obtained from study participants.

\section{Competing interests}

The authors declare that they have any competing interests for this article.

\section{Author details}

'Department of Orthopaedics, Auguste-Viktoria Clinic, Ruhr University Bochum, 32545 Bad Oeynhausen, Germany. ${ }^{2}$ Department of Orthopaedics, Trauma, and Reconstructive Surgery, RWTH University Hospital, Pauwelsstr. 30, 52074 Aachen, Germany.

Received: 25 January 2021 Accepted: 13 September 2021

Published online: 23 September 2021

\section{References}

1. Antomarchi J, Moeglin D, Laurichesse H, Combourieu D, Bigi N, Maisonneuve $E$, et al. The pubic diastasis measurement, a key element for the diagnosis, management, and prognosis of the bladder Exstrophy. Fetal Diagn Ther. 2019;45(6):435-40.

2. Lee EH, Shim JY. New sonographic finding for the prenatal diagnosis of bladder exstrophy: a case report. Ultrasound Obstet Gynecol. 2003;21(5):498-500.

3. Ebert AK, Reutter H, Ludwig M, Rosch WH. The exstrophy-epispadias complex. Orphanet J Rare Dis. 2009;4:23.

4. Kusakabe H, Ueoka K, Takayama S, Seki A. Gradual bone transfer for the correction of the pubic diastasis using the llizarov technique in closure of bladder and cloacal exstrophy. J Orthop Sci. 2018;23(1):144-50.

5. Suson KD, Sponseller PD, Gearhart JP. Bony abnormalities in classic bladder exstrophy: the urologist's perspective. J Pediatr Urol. 2013;9(2):112-22.

6. Yazici M, Kandemir U, Atilla B, Eryilmaz M. Rotational profile of lower extremities in bladder exstrophy patients with unapproximated pelvis: a clinical and radiologic study in children older than 7 years. J Pediatr Orthop. 1999;19(4):531-5.

7. Nordin S, Clementson C, Herrlin K, Hagglund G. Hip configuration and function in bladder exstrophy treated without pelvic osteotomy. J Pediatr Orthop B. 1996;5(2):119-22.

8. Nhan DT, Sponseller PD. Bilateral anterior innominate osteotomy for bladder Exstrophy. JBJS Essent Surg Tech. 2019;9(1):e1.

9. Stec AA, Pannu HK, Tadros YE, Sponseller PD, Wakim A, Fishman EK, et al. Evaluation of the bony pelvis in classic bladder exstrophy by using 3D-CT: further insights. Urology. 2001;58(6):1030-5.

10. Sponseller PD, Bisson L, Gearhart JP, Jeffs RD, Magid D, Fishman E. The anatomy of the pelvis in the exstrophy complex. J Bone Joint Surg Am. 1995:77(2):177-89.

11. Jeffs RD, Guice $S L$, Oesch I. The factors in successful exstrophy closure. J Urol. 1982;127(5):974-6.

12. Silver RI, Sponseller PD, Gearhart JP. Staged closure of the pelvis in cloacal exstrophy: first description of a new approach. J Urol. 1999;161 (1):263-6.

13. Mathews R, Gearhart JP, Bhatnagar R, Sponseller P. Staged pelvic closure of extreme pubic diastasis in the exstrophy-epispadias complex. J Urol. 2006;176(5):2196-8.

14. McKenna PH, Khoury AE, McLorie GA, Churchill BM, Babyn PB, Wedge JH. Iliac osteotomy: a model to compare the options in bladder and cloacal exstrophy reconstruction. J Urol. 1994;151(1):182-6 discussion 186-187.

15. Drobniewski M, Borowski A, Krasmska M, Sibmski M, Synder M. Hip joint arthroplasty in pubic symphysis separation in the course of congenital bladder exstrophy - study of two cases. Ortop Traumatol Rehabil. 2016;18(4):367-73.

16. Camera A, Grappiolo G, Santoro G. Hip arthroplasty in a patient with congenital pubic diastasis and bladder exstrophy. Hip Int. 2009;19(2):155-6.

17. Kovalenko B, Bremjit P, Fernando N. Classifications in brief: Tonnis classification of hip osteoarthritis. Clin Orthop Relat Res. 2018;476(8):1680-4.

18. Crowe JF, Mani VJ, Ranawat CS. Total hip replacement in congenital dislocation and dysplasia of the hip. J Bone Joint Surg Am. 1979;61(1):15-23.

19. Harris WH. Traumatic arthritis of the hip after dislocation and acetabular fractures: treatment by mold arthroplasty. An end-result study using a new method of result evaluation. J Bone Joint Surg Am. 1969;51(4):737-55.

20. Sponseller PD, Jani MM, Jeffs RD, Gearhart JP. Anterior innominate osteotomy in repair of bladder exstrophy. J Bone Joint Surg Am. 2001;83(2):184-93.

21. Imai H, Miyawaki J, Kamada T, Takeba J, Mashima N, Miura H. Preoperative planning and postoperative evaluation of total hip arthroplasty that takes combined anteversion. Eur J Orthop Surg Traumatol. 2016;26(5):493-500.

22. Laaksonen I, Lorimer M, Gromov K, Eskelinen A, Rolfson O, Graves SE, et al. Trabecular metal acetabular components in primary total hip arthroplasty. Acta Orthop. 2018;89(3):259-64.

23. Zhang $Y$, Ahn P, Fitzpatrick D, Heiner AD, Poggie R, Brown T. Interfacial frictional behavior: Cancellous bone, cortical bone, and a novel porous tantalum biomaterial. J Musculoskelet Res. 1999:3:245-51.

24. Perticarini L, Zanon G, Rossi SM, Benazzo FM. Clinical and radiographic outcomes of a trabecular titanium acetabular component in hip arthroplasty: results at minimum 5 years follow-up. BMC Musculoskelet Disord. 2015;16:375.

\section{Publisher's Note}

Springer Nature remains neutral with regard to jurisdictional claims in published maps and institutional affiliations.

Ready to submit your research? Choose BMC and benefit from:

- fast, convenient online submission

- thorough peer review by experienced researchers in your field

- rapid publication on acceptance

- support for research data, including large and complex data types

- gold Open Access which fosters wider collaboration and increased citations

- maximum visibility for your research: over $100 \mathrm{M}$ website views per year

At BMC, research is always in progress.

Learn more biomedcentral.com/submissions 